\title{
Comparing Linear Parameter-Varying Gain-Scheduled Control Techniques for Active Flutter Suppression
}

\author{
Jeffrey M. Barker* and Gary J. Balas ${ }^{\dagger}$ \\ University of Minnesota, Minneapolis, Minnesota 55455
}

\begin{abstract}
Two linear parameter-varying gain-scheduled controllers for active flutter suppression of the NASA Langley Research Center's Benchmark Active Control Technology (BACT) wing section are presented and compared to a previously presented gain-scheduled controller. The BACT wing section changes significantly as a function of Mach and dynamic pressure. The two linear parameter-varying (LPV) gain-scheduled controllers incorporate these changes as well as bounds on the rate of change of Mach and dynamic pressure. The inclusion of rate bounds in the design process allows for improved performance over a larger range of operating conditions than previously achieved by a linear fractional transformation gain-scheduled controller. The LPV controllers differ in that one primarily reduces coupling between the trailing-edge flap and the pitch and plunge modes, whereas the second optimizes wind gust attenuation. Closed-loop stability and improved performance are demonstrated via time simulations in which both Mach and dynamic pressure are allowed to vary in the presence of a Dryden wind-gust disturbance.
\end{abstract}

\section{Nomenclature}

\begin{tabular}{|c|c|}
\hline$A, B, C, D$ & $=$ state-space representation of a system \\
\hline$F_{u}, F_{l}$ & $=$ upper and lower linear fractional transformations \\
\hline & $=$ identity matrix \\
\hline$K_{\mathrm{LFT}}$ & $=$ linear fractional transformation (LFT) controller \\
\hline$K 1_{\mathrm{LPV}}$ & $=$ linear parameter-varying $(\mathrm{LPV})$ controller 1 \\
\hline$K 2_{\mathrm{LPV}}$ & $=\mathrm{LPV}$ controller 2 \\
\hline$M$ & $=$ Mach number \\
\hline$N$ & $=$ constant $2 \times 2$ block matrix \\
\hline $\boldsymbol{P}$ & $=$ parameter-dependentplant \\
\hline $\bar{q}$ & $=$ dynamic pressure, $\mathrm{kPa}$ \\
\hline$u$ & $=$ control signal \\
\hline$y$ & $=$ measured plant variables \\
\hline$\Delta$ & $=$ uncertainty model \\
\hline$\delta_{M}$ & $=$ normalized variation in Mach \\
\hline$\delta_{\bar{q}}^{M}$ & $=$ normalized variation in dynamic pressure \\
\hline & $=$ structured singular value \\
\hline
\end{tabular}

Subscripts

\begin{tabular}{|c|c|}
\hline act & $=$ actuator \\
\hline$d$ & $=$ disturbance \\
\hline & $=$ input (to plant) multiplicative \\
\hline & $\begin{array}{l}=\text { controller parameter } \\
=\text { noise }\end{array}$ \\
\hline & $=$ performance \\
\hline
\end{tabular}

\section{Introduction}

$\mathbf{F}$ LUTTER is a dynamic instability resulting from the coupling of aerodynamic, elastic, and inertial forces that can result in sudden mechanical failure of an aircraft wing during flight. The phemomena was noticed as early as 1916 by German aeronautical engineers ${ }^{1}$ and characterized by Theodorsen in $1935 .^{2}$ Because of the severity of the potential problem, todays aircraft typically operate in a region well below the flutter boundary.

As new lightweight materials are incorporated into aircraft designs in efforts to save money and increase performance, active

Received 17 May 1999; revision received 10 February 2000; accepted for publication 3 March 2000. Copyright (C) 2000 by Jeffrey M. Barker and Gary J. Balas. Published by the American Institute of Aeronautics and Astronautics, Inc., with permission.

* Graduate Research Assistant, Department of Aerospace Engineering and Mechanics. Member AIAA.

${ }^{\dagger}$ Associate Professor, Department of Aerospace Engineering and Mechanics. Member AIAA. flutter suppression will become important. The NASA Langley Research Center's Benchmark Active Control Technology (BACT) program was developed specifically to address the flutter problem., A description of the BACT model and aerodynamic data are given in Ref. 4. The BACT model has been used to design and test many types of flutter suppression control strategies including adaptive neural control, ${ }^{5,6}$ general predictive control, ${ }^{7}$ robust passification, ${ }^{8}$ robust multivariable control, ${ }^{9}$ and optimal control. ${ }^{10}$

This paper focuses on the design and comparison of linear parameter-varying (LPV) gain-scheduled controllers with a previously presented ${ }^{11}$ linear fractional transformation (LFT) gainscheduled controller. Gain-scheduled LPV control is a natural extension of $H_{\infty}$ control for systems that vary smoothly as a function of the chosen scheduling parameters. LPV theory offers advantages over classical gain-scheduled control in that the resulting LPV controllers are automatically gain scheduled, and no ad hoc methods of gain-schedulingare needed. Additionally,LPV gain-scheduledcontrol theory offers robustness guarantees that more traditional methods of gain scheduling cannot supply. Finally, LPV gain-scheduled control theory also has the advantage of explicitly incorporating bounds on the rate of change of the scheduling parameters. These rate bounds allow the design of less conservative controllers than those obtainablethrough gain-scheduledLFT control.For the BACT model, theoretical models at various dynamic pressures and Mach numbers were developed that correspond well to experiments at the same operating conditions. Thus, it is natural to choose to schedule an LPV controller on Mach and dynamic pressure. The LPV gainscheduled controllers presented have a designed operating range over the entire range of linear models considered, from Mach 0.5 to 0.82 and dynamic pressures from 3.59 to $10.77 \mathrm{kPa}$. The previously described gain-scheduledLFT control law has a restricted operating range (Mach 0.5-0.82 and 6.5-10.77 $\mathrm{kPa}$ ) and displays comparatively poor performance outside that operating range. Comparison of the controllers performance and a discussion of the apparent merits and deficiencies of LPV and LFT gain-scheduled control will form the bulk of the paper.

This paper is presented in the following seven sections. Section II describes the BACT facility, the wing section testbed, and the linear, time-invariant (LTI) models derived at specific Mach numbers and dynamic pressures. In Sec. III, a short description of LFT gain-scheduled theory and the previously presented LFT controller is given. Section IV presents the control theory associated with LPV gain-scheduled control. Control design and synthesis of the LPV gain-scheduled controllers are presented in Sec. V. In Sec. VI, the LPV control laws are analyzed and simulated, and a summary of the LFT gain-scheduled controller's performance is given. The 
Table 1 Flight conditions of LTI models

\begin{tabular}{lllllllll}
\hline \hline Mach & \multicolumn{7}{c}{ Dynamic pressure, kPa } \\
\hline 0.50 & 3.59 & 4.79 & 5.84 & $6.32^{\mathrm{a}}$ & $7.18^{\mathrm{a}}$ & $8.38^{\mathrm{a}}$ & $9.58^{\mathrm{a}}$ & $10.77^{\mathrm{a}}$ \\
0.70 & 3.59 & 4.79 & 5.99 & 6.51 & $6.99^{\mathrm{a}}$ & $8.38^{\mathrm{a}}$ & $9.58^{\mathrm{a}}$ & $10.77^{\mathrm{a}}$ \\
0.78 & 3.59 & 4.79 & 5.99 & 6.75 & $7.22^{\mathrm{a}}$ & $8.38^{\mathrm{a}}$ & $9.58^{\mathrm{a}}$ & $10.77^{\mathrm{a}}$ \\
0.82 & 3.59 & 4.79 & 5.99 & 6.84 & $7.33^{\mathrm{a}}$ & $8.38^{\mathrm{a}}$ & $9.58^{\mathrm{a}}$ & $10.77^{\mathrm{a}}$ \\
\hline \hline
\end{tabular}

${ }^{\mathrm{a} O p e n-l o o p ~ u n s t a b l e . ~}$

Flap to TE and LE acceleration Dryden gust to TE and LE acceleration
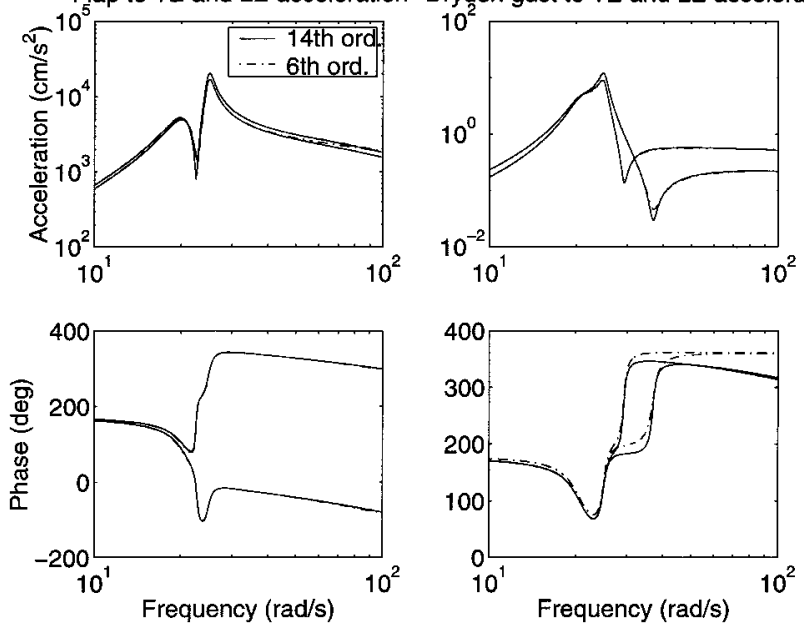

Fig. 1 Full- and reduced-order transfer function models from TE flap and wind gust to TE and LE accelerometers at $\bar{q}=8.38 \mathrm{kPa}$ and Mach $=0.7$.

stability and performance of all three gain-scheduledcontrollers are evaluatedover varying $M$ and $\bar{q}$. These results and the relative merits of LPV and LFT gain-scheduled control are discussed in Sec. VII. The final section summarizes the results and presents conclusions.

\section{BACT Model}

The BACT model is an element of NASA Langley Research Center's Benchmark Models Program, ${ }^{3}$ which consists of several models used to investigate aeroelastic effects and acquire experimental data for the validation of the computational fluid dynamic code. An overview of the BACT program, a description of the wind-tunnel model, and a summary of results are given in Ref. 4.

The experimentally validated theoretical LTI models used (Table 1) operate at four Mach numbers and range in dynamic pressure from 3.59 to $10.77 \mathrm{kPa}$. These LTI models have one control input (the trailing-edge flap) and leading- and trailing-edge acceleration measurements. The LTI models have 14 states: 4 to represent the pitch and plunge dynamics, 6 states that characterize unsteady aerodynamics, 2 states for the actuator dynamics, and the final 2 states are a second-order Dryden turbulence model.

Bode plots of the BACT theoretical LTI models indicate that they are primarily functions of the six states representing pitch, plunge, and actuator dynamics. The model states due to unsteady aerodynamics have a small contribution to the overall system response as do the gust model states. Figure 1 shows representative magnitude plots for the full- (14th-) order and residualized- (6th-) order LTI systems. Such a residualization leaves the state order intact, which is essential to the application of LPV gain-scheduledcontrol theory. The reduced-ordersystems appear to represent the physical dynamics of the BACT wing quite well. The relatively small effect of the unsteady aerodynamics is not surprising because the wing was designed so that the aerodynamic instabilities would be relatively benign. This attribute makes it easier to build in safety mechanisms to the wing section. ${ }^{12}$

The 32 LTI models used in this study (Table 1), thus, have six states, two states correspondingto each of pitch, plunge, and actuator dynamics. The models have two inputs and two outputs. The inputs are the command to the trailing-edge flap actuator and the input to the Dryden gust model. The outputs are leading- and trailing-edge acceleration measurements.

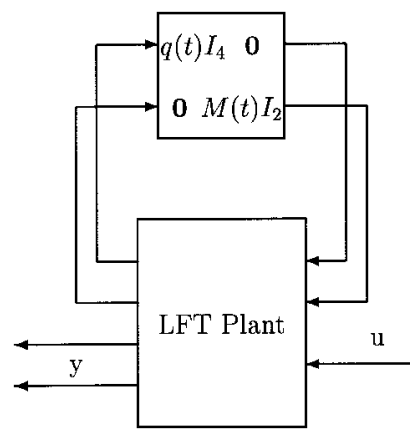

Fig. 2 LFT plant as a function of $\bar{q}$ and $M$.

\section{LFT Gain-Scheduled Control}

A gain-scheduled LFT control law for active flutter supression of the BACT wing was presented previously. ${ }^{11}$ A brief summary of gain-scheduled LFT control theory and the resulting controller is presented to allow for a more meaningful comparison between the previous results and the performance attained by the LPV gainscheduled controllers.

The central idea of gain-scheduled LFT control is that an openloop plant can often be represented as a linear fractional transformation of a nominal plant and physical parameters that vary within known bounds. If these parameters can be measured in real time, a controller can be synthesized that schedules as a function of these parameters. The gain-scheduled LFT framework as applied to the BACT model requires that the open-loop plant models for the operating range of interest be reformulated as a single open-loop model that varies as an LFT in the parameters $\bar{q}$ and $M$. These uncertain parameters are then separated out from the constant terms of the open-loop plant resulting in the interconnection structure show in Fig. 2. In Ref. 11, four copies of the parameter $\bar{q}$ and two copies of the parameter $M$ were necessary to represent adequately the variations of the model as a function of Mach number and dynamic pressure. A simple LFT is used to transform Fig. 2 into the standard $\mu$-synthesis form, such that the nominal plant model is represented by an interconnection of a constant system with a block diagonal uncertainty matrix whose parameters vary between -1 and 1 .

Consider a parameter-dependent plant modeled as an LFT of a time-varying block diagonal matrix $\Delta(t)$ and a three input/three output LTI plant $\boldsymbol{P}$. The parameter dependence of the system is due entirely to the time-varying $\Delta$ matrix $\Delta(t)$. For this flutter problem, the $\Delta(t)$ block is

$$
\Delta(t)=\left[\begin{array}{cc}
\delta_{\bar{q}}(t) I_{4} & 0 \\
0 & \delta_{M}(t) I_{2}
\end{array}\right]
$$

It is assumed that $\Delta(t)$ takes values in a known set $\Delta, \Delta(t)$ can be measured in real time, and $\Delta(t)$ varies between -1 and 1 .

The LFT parameter-dependent controller is restricted to have a structure similar to that of the plant. By interconnecting the parameter-dependent plant and controller, the closed-loop system appears as a finite-dimensional LTI system subjected to the timevarying perturbation $\Delta(t)$. The perturbation has a structure consisting of two parts: the physical parameters that affect the plant and the measured parameters that are used by the controller. In the development of the theory, the measured parameters are assumed to match exactly the physical parameters and are measured in real time. ${ }^{13}$

The control objective is to design the controller $K_{\mathrm{LFT}}$ such that for all allowable perturbations $\Delta(t) \in \Delta$ the parameter-dependent closed-loop system is internally exponentially stable with small induced $\mathcal{L}_{2}$ norm from disturbances to errors, including measurement noise and bounds on control authority. The small-gain theorem can be employed to bound (conservatively) the stability of the system and the induced $\mathcal{L}_{2}$ norm of the disturbance to error channels of the parameter-dependent closed-loop system. Because the perturbations $\Delta(t)$ have special repeated and block diagonal sturcture, the conservatism of the small-gain theorem may be reduced via input/ output similarity scalings. These scaling matrices are restricted to be constant diagonal matrices so that they will commute with the repeated structure of the perturbation. The main result of this formulation is that existence of a controller satisfying the scaled small-gain bound can be expressed exactly as the feasibility of a 
finite-dimensionalaffine matrix inequality ${ }^{14}$ (AMI). Because of the convexity of the AMI, this problem can be computed numerically. The details of gain-scheduled LFT control theory are covered in detail elsewhere. ${ }^{13,15}$

The gain-scheduledLFT control law previously presented has an operating range from 6.5 to $10.77 \mathrm{kPa}$ and from Mach 0.5 to 0.82 . The controller improves performance in comparison to the openloop system and a $\mu$-synthesis controller designed for the same open-loop plant. ${ }^{11}$

\section{LPV Gain-Scheduled Control}

A brief overview of LPV gain-scheduled control theory is given. Consider an $n$ th-order LPV system, whose dynamics evolve as

$$
\begin{aligned}
{\left[\begin{array}{c}
\dot{x}(t) \\
e_{1}(t) \\
e_{2}(t) \\
y(t)
\end{array}\right] } \\
\quad=\left[\begin{array}{cccc}
A[\rho(t)] & B_{11}[\rho(t)] & B_{12}[\rho(t)] & B_{2}[\rho(t)] \\
C_{11}[\rho(t)] & 0 & 0 & 0 \\
C_{12}[\rho(t)] & 0 & 0 & I_{n_{u}} \\
C_{2}[\rho(t)] & 0 & I_{n_{y}} & 0
\end{array}\right]\left[\begin{array}{c}
x(t) \\
d_{1}(t) \\
d_{2}(t) \\
u(t)
\end{array}\right]
\end{aligned}
$$

where $\rho(t)$ is piecewise continuous in a known compact set $\mathcal{P}$ and $|\dot{\rho}| \leq v$ (Ref. 16). The LPV gain-scheduled controller will depend explicitly on the parameters $\rho(t)$ and guarantee that stability and performance requirements are met for all allowable trajectories of $\rho(t)$ in some known, bounded set. Performance is measured via the induced $\mathcal{L}_{2}$ norm of a quadratically stable LPV system $G$ with zero initial conditions, defined as

$$
\|G\| \doteq \sup _{\rho} \sup _{\substack{\|d\|_{2} \neq 0 \\ d \in \mathcal{C}_{2}}} \frac{\|e\|_{2}}{\|d\|_{2}}
$$

Thus, the quadratic LPV $\gamma$-performance problem is to find an LPV controller of the form

$$
\left[\begin{array}{c}
\dot{x}_{K}(t) \\
u(t)
\end{array}\right]=\left[\begin{array}{ll}
\boldsymbol{A}_{K}[\rho(t)] & \boldsymbol{B}_{K}[\rho(t)] \\
\boldsymbol{C}_{K}[\rho(t)] & \boldsymbol{D}_{K}[\rho(t)]
\end{array}\right]\left[\begin{array}{c}
x_{K}(t) \\
y(t)
\end{array}\right]
$$

that minimizes the closed-loop $\mathcal{L}_{2}$ norm of the LPV system. If there exists a controller such that the closed-loop system is quadratically stable and the induced $\mathcal{L}_{2}$ norm from $d$ to $e$, as defined in Eq. (2), is less than $\gamma$, then the quadratic LPV $\gamma$-performance problem is solvable. The existence of a controller that solves the LPV problem can be expressed as the feasibility of the set of linear matrix inequalities (LMIs):
If the conditions of the preceding LMIs are satisfied, then by continuity and compactness, perturb $X$ such that the LMIs still hold and $\left(X-Y^{-1}\right)>0$ uniformly on $\mathcal{P}$. Then an $n$-dimensional strictly proper controller that solves the feedback problem is defined as

$$
\begin{aligned}
& A_{K}(\rho, \dot{\rho}):=A(\rho)+\gamma^{-1}\left[Q^{-1}(\rho) X(\rho) L(\rho) B_{12}^{T}(\rho)\right. \\
& \left.\quad+B_{1}(\rho) B_{1}^{T}(\rho)\right] Y^{-1}(\rho)+B_{2}(\rho) F(\rho) \\
& \quad+Q^{-1}(\rho) X(\rho) L(\rho) C_{2}(\rho)-Q^{-1}(\rho) H(\rho, \dot{\rho}) \\
& B_{K}(\rho):=-Q^{-1}(\rho) X(\rho) L(\rho) \\
& C_{K}(\rho):=F(\rho)
\end{aligned}
$$

where

$$
\begin{aligned}
Q(\rho) & :=X(\rho)-Y(\rho)^{-1} \\
F(\rho) & :=-\left[\gamma B_{2}^{T}(\rho) Y^{-1}(\rho)+C_{12}(\rho)\right] \\
L(\rho) & :=-\left[\gamma X^{-1}(\rho) C_{2}^{T}(\rho)+B_{12}(\rho)\right] \\
H(\rho, \dot{\rho}) & :=-\left[A_{F}^{T}(\rho) Y^{-1}(\rho)+Y^{-1}(\rho) A_{F}(\rho)+\sum_{i}\left(\dot{\rho}_{i} \frac{\partial Y^{-1}}{\partial \rho_{i}}\right)\right. \\
& \left.+\gamma^{-1} C_{F}^{T}(\rho) C_{F}(\rho)+\gamma^{-1} Y^{-1}(\rho) B_{1}(\rho) B_{1}^{T}(\rho) Y^{-1}(\rho)\right]
\end{aligned}
$$

with

$$
\begin{aligned}
& A_{F}(\rho):=A(\rho)+B_{2}(\rho) F(\rho) \\
& C_{F}(\rho):=\left[\begin{array}{c}
C_{11}(\rho) \\
C_{12}(\rho)+F^{T}(\rho)
\end{array}\right]
\end{aligned}
$$

For more details on LPV synthesis results, the reader is referred elsewhere. ${ }^{17-20}$ The parameter $\rho$ is assumed to be available in real time, and hence, it is possible to construct an LPV controller whose dynamics adjust according to variations in $\rho$ and maintain stability and performance along all parameter trajectories.

This approach allows gain-scheduled controllers to be treated as a single entity, with the gain scheduling achieved via the parameterdependent controller. This allows for a simple implementation of the LPV controller, linear interpolation between the corresponding parameter-dependent state-space controller matrices, provided the parameter dependence is sufficiently smooth between gridpoints. This approach has been successfully applied to the synthesis of

$$
\begin{aligned}
& {\left[\begin{array}{ccc}
Y(\rho) \hat{A}^{T}(\rho)+\hat{A}(\rho) Y(\rho)-\sum_{i} \pm\left(v_{i} \frac{\partial Y}{\partial \rho_{i}}\right)-\gamma B_{2}(\rho) B_{2}{ }^{T}(\rho) & Y(\rho) C_{11}^{T}(\rho) & B_{1}(\rho) \\
C_{11}(\rho) Y(\rho) & -\gamma I_{n_{e 1}} & 0 \\
B_{1}^{T}(\rho) & 0 & -\gamma I_{n_{d}}
\end{array}\right]<0} \\
& {\left[\begin{array}{c}
\tilde{A}^{T}(\rho) X(\rho)+X(\rho) \tilde{A}(\rho)+\sum_{i} \pm\left(v_{i} \frac{\partial X}{\partial \rho_{i}}\right)-\gamma C_{2}^{T}(\rho) C_{2}(\rho) \\
B_{11}^{T}(\rho) X(\rho) \\
C_{1}(\rho)
\end{array}\right.} \\
& \left.\begin{array}{cc}
X(\rho) B_{11}(\rho) & C_{1}^{T}(\rho) \\
-\gamma I_{n_{d 1}} & 0 \\
0 & -\gamma I_{n_{e}}
\end{array}\right]<0, \\
& {\left[\begin{array}{cc}
X(\rho) & I_{n} \\
I_{n} & Y(\rho)
\end{array}\right] \geq 0}
\end{aligned}
$$

where $X(\rho)$ and $Y(\rho)$ are continuously differentiable real matrix functions such that $X(\rho)>0, Y(\rho)>0$, and

$$
\begin{array}{ccc}
\hat{A}(\rho):=A(\rho)-B_{2}(\rho) C_{12}(\rho), & B_{1}(\rho)=\left[\begin{array}{ll}
B 11(\rho) & B 12(\rho)
\end{array}\right] \\
\tilde{A}(\rho):=A(\rho)-B_{12}(\rho) C_{2}(\rho), & C_{1}^{T}=\left[\begin{array}{ll}
C_{11}^{T}(\rho) & C_{12}^{T}(\rho)
\end{array}\right]
\end{array}
$$

missile autopilots ${ }^{21}$ controllers for turbofan engines, ${ }^{22}$ and flight controllers. ${ }^{23}$

\section{LPV Control Design and Synthesis}

The control design methodology is very similar to that of $H_{\infty}$ control design. Both LPV gain-scheduledcontrol laws are designed 


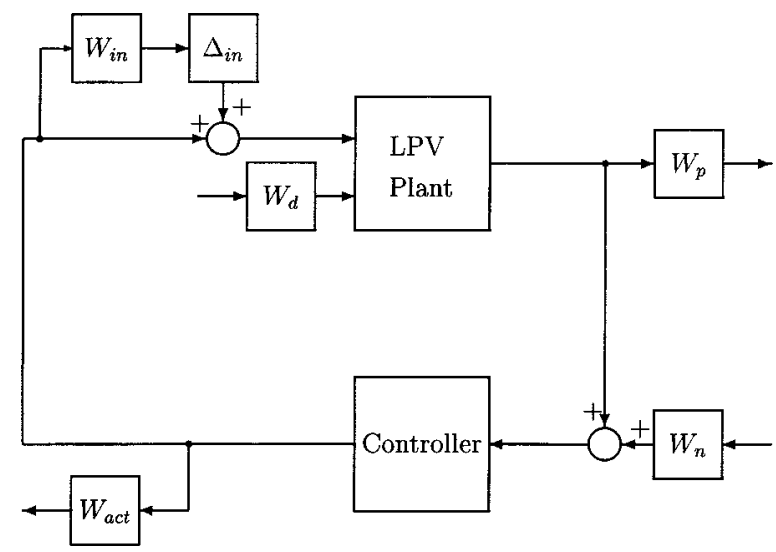

Fig. 3 LPV control design block diagram.

in Sec. V.A using the same block diagram and most of the same weighting functions. As with the standard $H_{\infty}$ control algorithms, the resulting controllers have the same state order as the weighted open-loop plants. Controller synthesis involves solutions of LMIs and is discussed in Sec. V.B.

\section{A. Control Design}

The reduced six-state LFT plant (four aerodynamic states and two actuator states) is used to design both LPV flutter suppression controllers. This reduced plant has two inputs and two outputs. The first input is flap position, in radians per second (the control input), and the second is an input to the Drygden wind-gust model. The two outputs are trailing-and leading-edgeaccelerationin centimetersper second squared, and both are used for feedback control.

The block diagram in Fig. 3 is used in the synthesis of both of the LPV gain-scheduled controllers. This diagram corresponds to the integration of performance objectives and robust stability objectives into a single control design framework. The stability objectives are to stabilize the wing throughoutthe operating region and to be robust to uncertainty in the modeling process and to errors introduced by the model reduction process. These robustness objectives are incorporated via input multiplicative uncertainty. All 32 reduced-order LTI models of the BACT wing are used in the control synthesis algorithm. Performance requirements are formulated through the choice of the weighting functions applied to the input and output signals of the open-loop system. The objective is to achieve an induced $\mathcal{L}_{2}$ norm less than 1 with the resulting LPV controller. Thus, all objectives are scaled by weighting functions to be less than 1 when achieved.

The leading- and trailing-edge accelerations are the primary performance signals. A noise signal is added to the acceleration signals to corrupt the measurements. The flap command signal from the controller is also restricted to reflect the physical limitations on this actuator. A weighted Dryden wind-gust disturbance signal allows for unknown exogenous disturbances to enter the system. All of the elements of the block diagram, except for the disturbance weight, are chosen to be the same for both LPV control laws. Thus, the differences in the control laws are entirely due to the differences in the choice of disturbance weights.

Multiplicative uncertainty, represented in the block diagram by the weight $W_{\text {in }}$ and the uncertainty set $\Delta_{\text {in }}$, is used to capture modeling error at high frequency and uncertainty introduced by the model reduction process. For this system, the multiplicative uncertainty weight used is $W_{\text {in }}=0.1(s / 2+1) /(s / 200+1)$, representing $10 \%$ uncertainty in the LPV model at low frequencies, $100 \%$ uncertainty at $20 \mathrm{rad} / \mathrm{s}$, and $1000 \%$ at high frequencies. The level of uncertainty at high frequency ensures that the controllers will not amplify the system dynamics in this frequency range. This multiplicative uncertainty weight is held constant across the entire operating region.

One of the primary performance objectives for active flutter suppression is to limit oscillations of the wing at the flutter frequencies. This objective is captured via a constant diagonal performance weighting $W_{p}$, which restricts the maximum magnitude of the dis- turbance and actuator to acceleration transfer functions. First $W_{p}$ is chosen to normalize the trailing-edge flap to acceleration output channels (in centimeters per second squared per radian) to have a peak value of approximatelyone. (Approximately, because the peak values of the transfer functions from flap command to leading- and trailing-edge acceleration vary as functions of $\bar{q}$ and $M$, whereas $W_{p}$ is constant over the entire operating range.) These constants are then multiplied by 2 , asking that the peak magnitude be reduced to half of its initial value. Thus, for both LPV control laws,

$$
W_{p}=2\left[\begin{array}{cc}
1 / 25,000 & 0 \\
0 & 1 / 25,000
\end{array}\right]
$$

is chosen. These constant weights, applied to the trailing- and leading-edge acceleration output channels, request a reduction of the maximum singular values from the trailing-edge flap input to the acceleration outputs. Thus, these weights correspond to asking for a decrease to $50 \%$ of the open-loop peak response at the natural frequencies for the stable plants and to similar magnitudes at the flutter frequency for the open-loop unstable plants. Because the basic performance problem is one of vibration attenuation, the constant performance weight is all that is needed to suppress the peak singular values. Additionally, by choosing a constant performance weight, the order of the gain-scheduledLPV controllers is kept low. (A first-order performance weight on each output channel would add two states to the controller, for example.)

The trailing-edge flap used as the actuator has limits of $\pm 15 \mathrm{deg}$ or $\pi / 12 \mathrm{rad}$. As in the performance weight, the actuator weight $W_{\text {act }}=12 / \pi$ is chosen to scale the largest allowable actuator command to \pm 1 . No rate limits are imposed on the actuator in this control design formulation because the high-frequency gain of the multiplicative uncertainty effectively limits the bandwidth of the controller.

Sensor noise is added to the feedback signals to corrupt the measurements and to satisfy the LPV control algorithm used for design. The weight $W_{n}=\operatorname{diag}[250,250]$ was chosen so that the maximum noise to signal ratio is about $10 \%$ in the frequency range $10-50 \mathrm{rad} / \mathrm{s}$.

Disturbances are introduced through a weighted Dryden windgust disturbance input. The disturbance weight is chosen as $W_{d}=1$ for the first LPV gain-scheduledcontroller $K 1_{\mathrm{LPV}}$ and as $W_{d}=1800$ for the second gain-scheduledcontrol law, $K 2_{\mathrm{LPV}}$. The large disparity is due to the different design criteria reflected by the two LPV designs. The open-loop transfer functions from flap to acceleration are about 1800 times greater than the transfer functions from disturbance to acceleration. As a result, the choice $W_{d}=1$ corresponds to a performance requirement that flap commands not excite the wing. $K 2_{\mathrm{LPV}}$ is designed asking that both flap commands and disturbance inputs do not excite the wing. Thus, $K 1_{\mathrm{LPV}}$ attempts to stabilize the system and to add damping to the flap-to-acceleration transfer function whereas $K 2_{\mathrm{LPV}}$ attempts to stabilize the system and to add damping to both the flap-to-acceleration and the disturbanceto-acceleration transfer functions. This suggests that $K 1_{\mathrm{LPV}}$ will better attenuate the excitation of the wing due to flap dynamics, whereas $K 2_{\mathrm{LPV}}$ should provide more damping in the wind-gust-toacceleration channel.

Finally, rate of change bounds on parameter variation are chosen for control synthesis. The bounds chosen are $0.3 \mathrm{kPa} / \mathrm{s}$ on $\bar{q}$ and Mach $0.02 / \mathrm{s}$ on $M$. These rate of change bounds are slow enough to have a significant effect on the resulting control laws.

\section{B. Controller Synthesis}

Both LPV gain-scheduled controllers were synthesized using an algorithm that returns LTI controllers at each of 32 interpolated gridpoints. These gridpoints are taken at the four Mach values of Table 1 and dynamic pressures $3.59,4.79,5.99,6.51,7.18,8.38,9.58$, and $10.77 \mathrm{kPa}$. These dynamic pressures are chosen to make a rectangular grid for the LPV control design process. The LTI models in this grid were determined via linear interpolation in $\bar{q}$ between the two nearest existing LTI models. When $\bar{q}$ and $M$ fall between the interpolated gridpoints, a point by point linear interpolation among the four nearest controllers provides the appropriate control law for that point in the design space. Both $K 1_{\mathrm{LPV}}$ and $K 2_{\mathrm{LPV}}$ stabilize the 
open-loop plant over the full range of Mach numbers (0.5-0.82) and dynamic pressures (3.59-10.77 kPa). The LPV gain-scheduled controllers are synthesized using a formulation of the induced $\mathcal{L}_{2}$ problem as a linear objectiveminimization. ${ }^{13}$ The two resulting LPV controllers have the same number of states as the weighted openloop system. Thus, the controllers are of seventh order (sixth-order nominal plant and a first-order model uncertainty weight) and have $\gamma$ levels of about 1.1 for $K 1_{\mathrm{LPV}}$ and about 1.6 for $K 2_{\mathrm{LPV}}$.

\section{Results}

The primary objectives were to improve the disturbance rejection characteristics of the wing and to increase the range of operating conditions at which the wing is stable. Several performance characteristics are considered in examining the success of the LPV gain-scheduledcontrollers at meeting these goals and in comparing their success to that of the previously presented gain-scheduledLFT controller. First, the stability and performance of the closed-loop system is examined using the full-order LTI single-point models and the point controllers obtained by specifying constant dynamic pressure and Mach. For example, the closed-loop system at a given Mach number and dynamic pressure was formed by the full-order (14-state) LTI model at Mach 0.7 and $10.77 \mathrm{kPa}$ closed with $K 1_{\mathrm{LPV}}$, $K 2_{\mathrm{LPV}}$, and $K_{\mathrm{LFT}}$ operating at the same Mach and $\bar{q}$. Second, the Bode plots of the open- and closed-loop systems are compared. Finally, time simulations of the closed-loop response of the wing as Mach and dynamic pressure vary are used to examine the disturbance rejection characteristicsover the full operating range, as well as to demonstrate reasonable actuator usage.

The structure of the LPV problem guarantees stability at the gridpoints providing that a feasible solution to the problem-specific LMIs is found. As long as the model reduction does not introduce significant changes in the open-loop transfer functions, it is reasonable to expect that the LPV controller at a given operating point will stabilize the full-order open-loop model at the same operating point. For this flutter problem, the difference between the fulland reduced-order models is small, and both LPV gain-scheduled controllers do stabilize the full-order systems throughout the operating range (Mach from 0.5 to 0.82 and $\bar{q}$ from 3.59 to $10.77 \mathrm{kPa}$ ). However, the dynamics of the resulting open-loop systems closed with $K 1_{\mathrm{LPV}}$ and $K 2_{\mathrm{LPV}}$ are significantly different, and, thus, merit comment.

Figure 4 shows Bode plots at Mach 0.7 and $9.58 \mathrm{kPa}$ (representative plots) for disturbance to trailing-edge acceleration for $K 1_{\mathrm{LPV}}$ and $K 2_{\mathrm{LPV}}$. Clearly, $K 2_{\mathrm{LPV}}$ achieves higher damping (0.17 compared to 0.05 , for this example) of the flutter mode and has greater high-frequency gain and phase margins than $K 1_{\mathrm{LPV}} . K 1_{\mathrm{LPV}}$, however, rejects both high- and low-frequency disturbance inputs significantly better than $K 2_{\mathrm{LPV}}$ and has better low-frequency stability margins. Figure 5 shows typical closed-loop Bode plots of the actu-
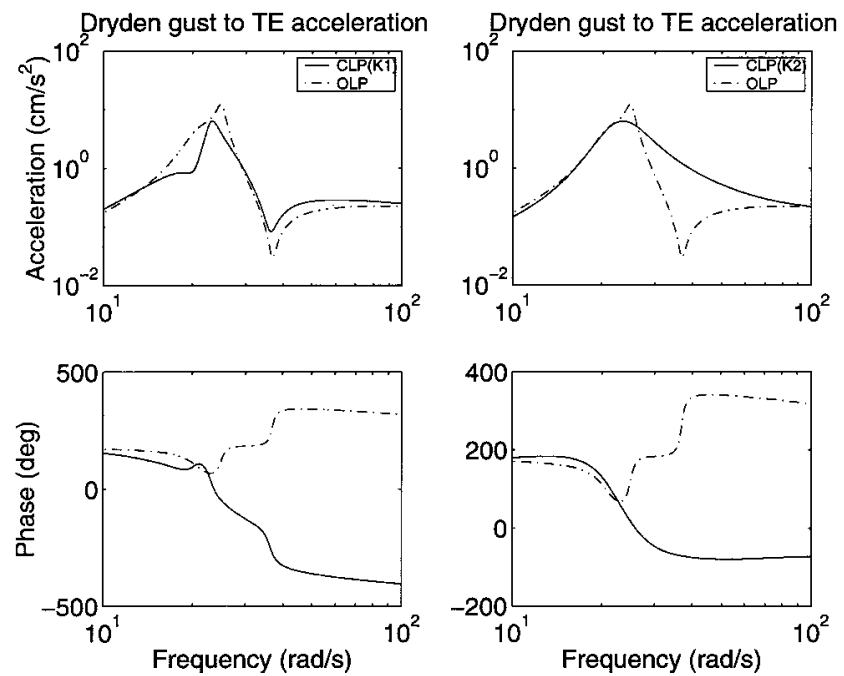

Fig. 4 Open- and closed-loop Bode plots from wind gust to TE acceleration for $K 1_{\mathrm{LPV}}$ and $K 2_{\mathrm{LPV}}$ at Mach 0.7 and $\bar{q}=9.58 \mathrm{kPa}$.
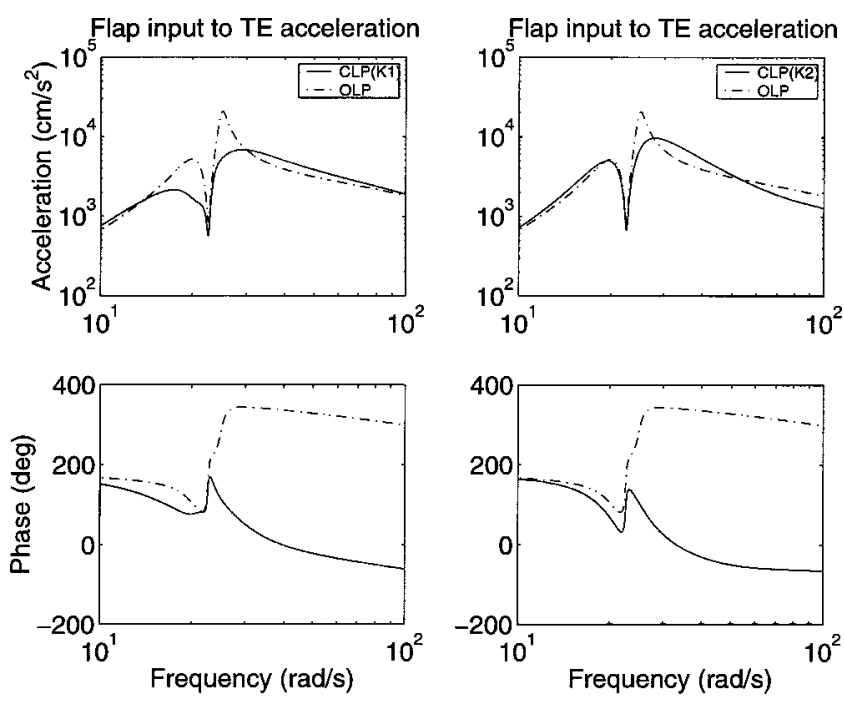

Fig. 5 Open- and closed-loop Bode plots from TE flap to TE acceleration for $K 1_{\mathrm{LPV}}$ and $K 2_{\mathrm{LPV}}$ at Mach 0.7 and $\bar{q}=9.58 \mathrm{kPa}$.
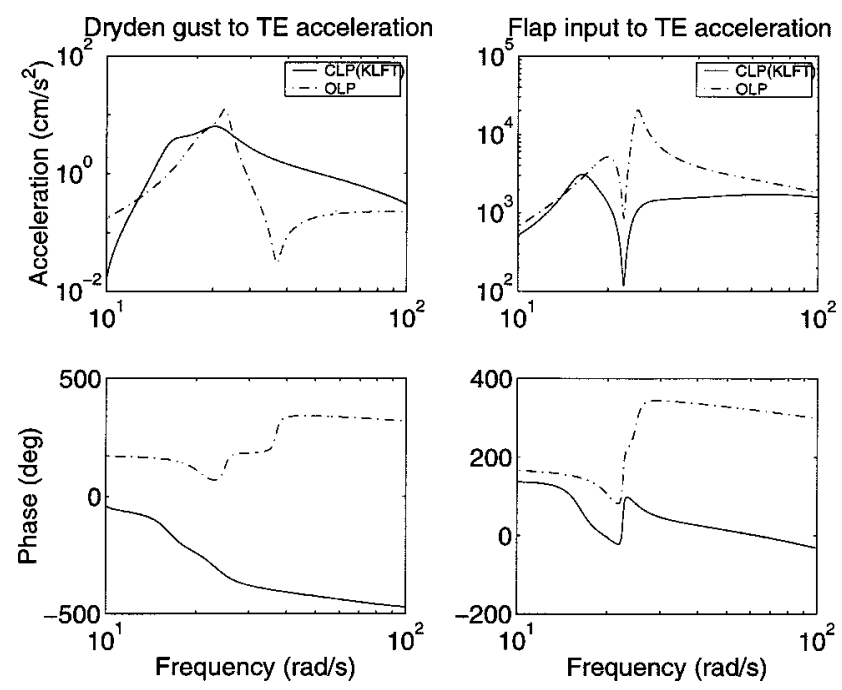

Fig. 6 Open- and closed-loop Bode plots from TE flap and wind gust to TE acceleration for $K_{\mathrm{LFT}}$ at Mach 0.7 and $\bar{q}=9.58 \mathrm{kPa}$.

ator to trailing-edge accelerations. Here, both closed-loop systems show significant increases in damping compared to the open-loop systems, but again the system closed with $K 1_{\mathrm{LPV}}$ appears to give better nominal performance (less coupling to acceleration) at the resonant frequencies of the wing. Thus, Figs. 4 and 5 show a distinct tradeoffbetween stability and performance. If the linear models are believed to be highly accurate, $K 1_{\mathrm{LPV}}$ would most likely be the preferred LPV gain-scheduled controller. If, however, the models are less well known at and above the flutter frequency, $K 2_{\mathrm{LPV}}$ is likely to be a more appropriate choice of control law. These results are directly correlated to the choice of the weight on the Dryden disturbance input in the control design process. $K 2_{\mathrm{LPV}}$, designed with a large weight on the input disturbance, appears to increase the damping of the flutter mode in the disturbance-to-accebration channels. $K 1_{\mathrm{LPV}}$, designed with a small input disturbance weight, focuses on the coupling between the trailing-edge flap and wing acceleration.

Similar plots for the previously presented $K_{\mathrm{LFT}}$ are revealing. Figure 6 shows that $K_{\mathrm{LFT}}$ more closely resembles $K 1_{\mathrm{LPV}}$ in eliminating the high-frequency flap-to-accelerationresonance. However, the right-hand plots in Fig. 6 demonstrate that the disturbance to trailing-edge (TE) acceleration channels are magnified except near the open-loop flutter frequency. These results reflect that $K_{\mathrm{LFT}}$ was designed without consideration of the Dryden wind-gustdisturbance effects. Redesigning with these effects included would likely result in closed-loop transfer functions similar to those of $K 2_{\mathrm{LPV}}$. 
Table 2 Time simulation results for three gain-scheduled control laws

\begin{tabular}{|c|c|c|c|c|c|c|c|c|}
\hline \multirow[b]{2}{*}{ Parameter } & \multicolumn{2}{|c|}{$\begin{array}{c}\text { TE acceleration, } \\
\mathrm{cm} / \mathrm{s}^{2}\end{array}$} & \multicolumn{2}{|c|}{$\begin{array}{c}\text { LE acceleration, } \\
\mathrm{cm} / \mathrm{s}^{2}\end{array}$} & \multicolumn{2}{|c|}{$\begin{array}{c}\text { Flap deflection, } \\
\text { deg }\end{array}$} & \multicolumn{2}{|c|}{$\begin{array}{c}\text { Flap rate, } \\
\text { deg/s }\end{array}$} \\
\hline & RMS & Peak & RMS & Peak & RMS & Peak & RMS & Peak \\
\hline$K 1_{\mathrm{LPV}}$ & 6.65 & 19.74 & 7.03 & 30.60 & 0.07 & 0.26 & 1.77 & 6.52 \\
\hline$K 2_{\mathrm{LPV}}$ & 9.27 & 29.30 & 9.37 & 33.61 & 0.07 & 0.27 & 2.37 & 11.91 \\
\hline$K_{\mathrm{LFT}}$ & 12.58 & 36.68 & 13.42 & 40.81 & 0.19 & 0.72 & 7.06 & 26.69 \\
\hline
\end{tabular}
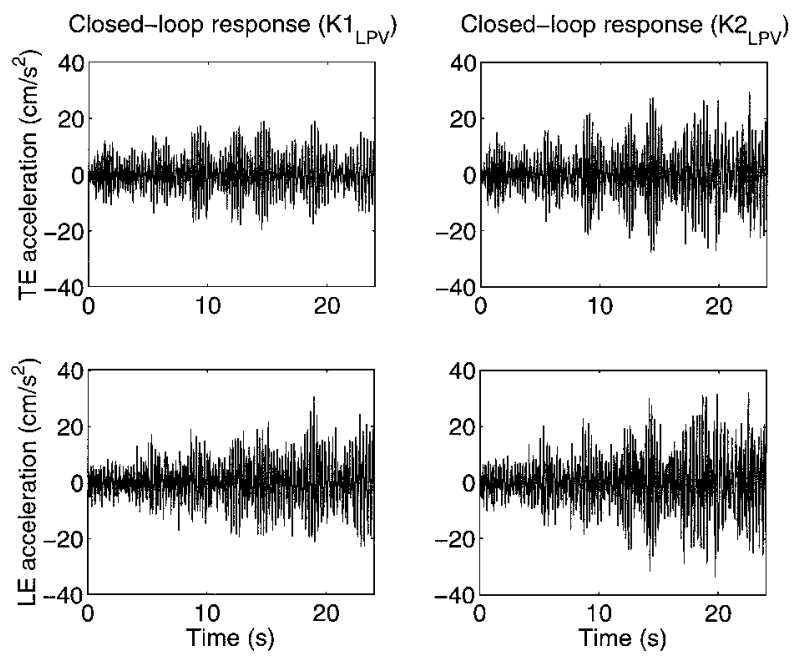

Fig. 7 Time response of TE and LE acceleration to wind-gust input for $K 1_{\mathrm{LPV}}$ and $K 2_{\mathrm{LPV}}$ as $M$ and $\bar{q}$ vary.
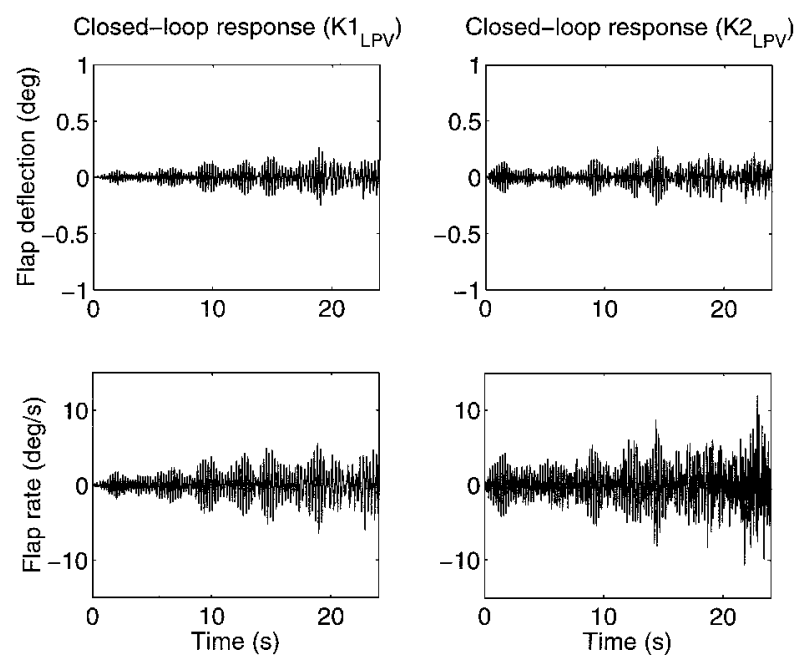

Fig. 8 Time response of TE flap deflection and rate to wind-gust input for $K 1_{\mathrm{LPV}}$ and $K 2_{\mathrm{LPV}}$ as $M$ and $\bar{q}$ vary.

Time simulations of the three closed-loop systems subject to an input distrubance while $M$ and $\bar{q}$ vary linearly over $25 \mathrm{~s}$ from Mach 0.5 to 0.82 and from 3.59 to $10.77 \mathrm{kPa}$ are presented to show stability and performance in the presence of wind gusts. The simulations are of the LPV and LFT controllersclosed with a full- (14th-) order LPV model of the BACT wing. The input disturbance is band-limited $(50-\mathrm{Hz})$ white noise to the Dryden wind-gust model. The constant offset of the white noise input is chosen so that the resulting wind gust has an average velocity of $2.54 \mathrm{~cm} / \mathrm{s}$. Note that the simulation enters the open-loop unstable operating region at approximately $9 \mathrm{~s}$. Figure 7 shows the resulting TE and leading-edge(LE) accelerations for the BACT wing with control laws $K 1_{\mathrm{LPV}}$ and $K 2_{\mathrm{LPV}}$. Figure 8 shows flap deflections and rates throughout the simulations for the BACT model closed with $K 1_{\mathrm{LPV}}$ and $K 2_{\mathrm{LPV}}$. These results show that $K 1_{\text {LPV }}$ outperforms $K 2_{\text {LPV }}$ while using less control energy. Figure 9
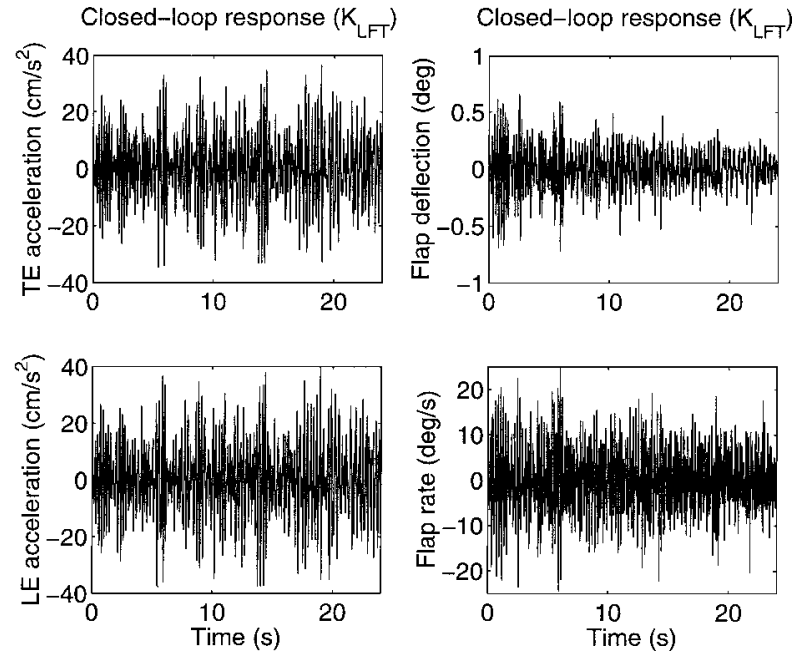

Fig. 9 Time response of $T E$ and $L E$ acceleration and control deflection and rate to wind gust for $K_{\mathrm{LFT}}$ as $M$ and $\bar{q}$ vary.

gives TE and LE accelerations and flap deflection and rate for the BACT model closed with $K_{\mathrm{LFT}}$. Figures 7-9 demonstrate that $K_{\mathrm{LFT}}$ achieves lower performance with larger and faster flap commands than that obtained by the LPV gain-scheduled controllers. The rms and peak TE and LE accelerations, flap deflections, and rates for the simulations of the BACT model closed with $K 1_{\mathrm{LPV}}, K 2_{\mathrm{LPV}}$, and $K_{\mathrm{LFT}}$ are given in Table 2 .

Examination of Table 2 and Figs. 7-9 reveal that in terms of minimizing the effects of input wind gusts on the TE and LE acceleration with a minimum of control effort the LPV gain-scheduled controllers outperform the gain-scheduledLFT controller. All three gain-scheduledcontrollersextend the flutter boundary to $10.77 \mathrm{kPa}$, the maximum dynamic pressure of the LTI models; hence, all three controllers can be considered successes. As a measurement of improvement of the LPV gain-scheduled control laws in comparison to the LFT controllaw, using the performance of the gain-scheduled LFT control law as a baseline is reasonable. $K 1_{\mathrm{LPV}}$ reduces peak and rms TE acceleration and rms LE acceleration by just less than $50 \%$, while reducing peak LE acceleration by about $25 \%$ in comparison to the performance achieved by $K_{\mathrm{LFT}}$. $K 2_{\mathrm{LPV}}$ reduces peak and rms TE and LE acceleration by about $20-30 \%$. More dramatic is actuator usage. For both $K 1_{\mathrm{LPV}}$ and $K 2_{\mathrm{LPV}}$, rms and peak flap deflections are $60 \%$ less than those of $K_{\mathrm{LFT}}$. Similarily, the $K 1_{\mathrm{LPV}}$ flap rates are $75 \%$ lower than the $K_{\mathrm{LFT}}$ flap rate, whereas the $K 2_{\mathrm{LPV}}$ flap rates are $60 \%$ lower.

\section{Discussion}

In this specific problem, LPV gain-scheduled control appears to offer significant performance advantages over gain-scheduled LFT control. Both LPV gain-scheduled controllers significantly reduce the effects of wind gusts and require smaller and less rapid actuation to achieve this improved performance. Although this cursory comparison of the results is certainly useful in this specific case, a more general discussion of the merits of these two different types of gain-scheduled control laws and how they apply to this problem is perhaps more important.

The primary advantages of the LPV gain-scheduled control methodology for this flutter example are the direct use of a grid of 
LTI models for LPV control design and the straightforward manner in which rate bounds on the time-varying parameters may be incorporated into the problem formulation. Another advantage of the LPV gain-scheduled control design method for this specific problem is the existence of LTI models in which the entries of the $\boldsymbol{A}$, $\boldsymbol{B}, \boldsymbol{C}$, and $\boldsymbol{D}$ matrices vary smoothly as a function of $\bar{q}$ and $M$. In other problems, including identified models of real systems, ensuring such smooth variance in the $\boldsymbol{A}, \boldsymbol{B}, \boldsymbol{C}$, and $\boldsymbol{D}$ matrices' entries is not as simple and often requires extra time be taken to set the systems up in the same manner.

To investigate further the importance of rate bounds on parameter variations to this specific problem, LPV control designs with very large rate bounds were attempted. These LPV designs were unable to stabilize the entire range (Table 1) of LTI models of the BACT wing. This shows that gain-scheduledLFT control's inability to incorporate rate bounds into the problem is a significant drawback for this flutter problem and that no gain-scheduledLFT controller could be synthesized over the entire 32 model operating range. For other problems, where the parameters under consideration can change very quickly, this inability to incorporaterate bounds is not a factor. One example of this situation would be creating a gain-scheduled LFT control law for a missile cruising at constant Mach, using $\alpha$ and $\beta$ as the scheduling parameters. Because the missile can change its angle of attack very quickly, introducing rate bounds into the problem may result in little or no improvement in performance.

LFT gain-scheduled control also has strong points. For flutter control of an airplane, accurate open-loop models above the flutter boundary are difficult to obtain and likely have large uncertainties. This type of problem may be more realistically approached in an LFT framework, where general trends of certain states or modes may be known, but not exact LTI models. For example, it may be known that a complex conjugate pole pair moves into the right halfplane while its natural frequency increases slowly. Such information can be used to formulate easily an LFT model of a problem, where a grid of LTI models may be difficult to generate.

Some of the drawbacks associated with the gain-scheduled LFT method as applied here could be eliminated. There are methods for reducing the conservatismof gain-scheduledLFT controlby switching from complex to real parameter variation. ${ }^{24,25}$ Also, because the gain-scheduledLFT control has exactly the same structure as $\mu$ synthesis, a form of gain-scheduled DK iteration with constant scaling matrices is possible, likely resulting in improved performance.

Finally, the testing and simulations were done using the fullorder LTI models, and the full-order LPV model consisting of the 32 interpolated gridpoints discussed in Sec. V.B. These are very similar to the models on which the LPV gain-scheduled control laws were based, differing only by the error introduced through the model-reduction process. The gain-scheduled LFT control design was based on an LFT model of the system that did not exactly match the LTI models. ${ }^{26}$ This further demonstrates that designing an LFT open-loop plant interconnection when high-quality LTI models are available will often be less effective than direct use of the grid of models via LPV gain-scheduled control.

\section{Conclusions}

Recently developed gain-scheduled control techniques in which the control laws are explicit functions of the scheduling parameters reduce design time when compared to ad hoc gain-scheduled control laws while yielding larger operating ranges (or improved performance over that range) in comparison to fixed-point control designs.

Rate-bounded LPV gain-scheduled control methods are used to design two gain-scheduled controllers for the entire range of models of the BACT wing. Both LPV controllers achieve increased performance (as measured by limiting TE and LE acceleration due to wind gusts) with smaller control surface deflections and rates compared to the gain-scheduled LFT controller. All three control laws discussed extend the flutter boundary to (past) the maximum $\bar{q}$ considered, $10.77 \mathrm{kPa}$. This represents a $60 \%$ increase in the flutter boundary as measured by dynamic pressure. In addition, the LPV gain-scheduled controllers significantly outperform the previously presented gain-scheduledLFT controller.
This specific flutter problem is formulated in a way that is readily adaptable to LPV gain-scheduled control techniques. The advantages and performance improvements achieved via LPV gainscheduled control in comparison to gain-scheduledLFT control are not indicative of a general result, but do point out some significant considerations. In cases where scheduling parameters may be expected to vary relatively slowly, such as this flutter example, LPV methodology has an advantage through easy incorporation of rate bounds into the problem structure. In cases where the scheduling parameters change on a much faster timescale, the improvements obtainable by introduction of rate bounds can be insignificant.

\section{Acknowledgments}

The authors wish to acknowledge the generous financial support from the Air Force Office of Scientific Research (AFOSR) under the Partnership for Research Excellence and Transition program (F49620-95-1-0419) and an AFOSR Augmentation Award for Science and Engineering Research Training Grant. The authors would also like to thank Carol Wieseman, NASA Langley Research Center, for allowing the use of the BACT data in our research.

\section{References}

${ }^{1}$ Kehoe, M. W., “A Historical Overview of Flight Flutter Testing,” Proceedings of Advanced Aeroservoelastic Testing and Data Analysis, CP-566, AGARD, 1995, pp. 1.1-1.15.

${ }^{2}$ Theodorsen, T., "General Theory of Aerodynamic Instability and the Mechanism of Flutter,” NACA 496, 1935.

${ }^{3}$ Bennet, R., Eckstrom, C., Rivera, J., Jr., Dansberry, B., Farmer, M., and Durham, M., "The Benchmark Aeroelastic Models Program-Description and Highlights of Initial Results," NASA TM-104180, 1991.

${ }^{4}$ Scott, R., Hoadley, S., Wieseman, C., and Durham, M., "Benchmark Active Controls Technology Model Aerodynamic Data," Journal of Guidance, Control, and Dynamics, Vol. 23, No. 5, 2000, pp. 915-922.

${ }^{5}$ Scott, R., Lichtenwainer, P., and Little, G., "Adaptive Neural Control of Aeroelastic Response," Journal of Guidance, Control, and Dynamics (to be published).

${ }^{6}$ Scott, R., Lichtenwainer, P., Pado, L., and Little, G., "Adaptive Neural Control for Active Flutter Suppression," Journal of Guidance, Control, and Dynamics (to be published).

${ }^{7}$ Haley, P., and Soloway, D., "The Benchmark Active Controls Technology Model Aerodynamic Database," Journal of Guidance, Control, and Dynamics (to be published).

${ }^{8}$ Kelkar, A., and Joshi, S., "Passivity-Based Robust Control with Application to Benchmark Active Controls Technology Wing," Journal of Guidance, Control, and Dynamics, Vol. 23, No. 5, 2000, pp. 938-947.

${ }^{9}$ Waszak, M., "Robust Multivariable Flutter Suppression for the Benchmark Active Control Technology (BACT) Wind-Tunnel Model," Journal of Guidance, Control, and Dynamics (to be published).

${ }^{10}$ Mukhopadhyay, V., "Transonic Flutter Suppression Control Law Design and Wind Tunnel Test Results," Journal of Guidance, Control, and Dynamics, Vol. 23, No. 5, 2000, pp. 930-937.

${ }^{11}$ Barker, J. M., Balas, G. J., and Blue, P. A., "Gain-Scheduled Linear Fractional Control for Active Flutter Suppression," Journal of Guidance, Control, and Dynamics, Vol. 22, No. 4, 1999, pp. 507-512.

${ }^{12}$ Haley, P., and Soloway, D., "Generalized Predictive Control for Active Flutter Suppression,’'IEEE Control Systems, Vol. 17, No. 4, 1997,pp. 64-70.

${ }^{13}$ Packard, A., "Gain Scheduling via Linear Fractional Transformations," Systems and Control Letters, Vol. 22, No. 2, 1994, pp. 79-92.

${ }^{14}$ Boyd, S., El Ghaoui, L., Feron, E., and Balakrishnan, V., Linear Matrix Inequalities in System and Control Theory, No. 15, Studies in Applied Mathematics, Society for Industrial and Applied Mathematics, Philadelphia, 1994.

${ }^{15}$ El Ghaoui, L., and Scorletti, G., "Control of Rational Systems Using Linear Fractional Representations and LMIs," IFAC Symposium on Robust Control, 1994.

${ }^{16}$ Kamen, E., and Khargonekar, P., "On the Control of Linerar Systems whose Coefficients are Functions of Parameters," IEEE Transactions on Automatic Control, Vol. 29, No. 1, 1984, pp. 25-33.

${ }^{17}$ Packard, A., and Kantner, M., "Gain Scheduling the LPV Way," Proceedings of the 35th IEEE Control Decision Conference, Inst. of Electrical and Electronics Engineers, New York, 1996, pp. 3938-3941.

${ }^{18}$ Becker, G., "Quadratic Stability and Performance of Linear Parameter Dependent Systems,” Ph.D. Thesis, Dept. of Mechanical Engineering, Univ. of California, Berkeley, CA, 1993.

${ }^{19}$ Sharuz, S. M., and Behtash, S., "Design of Controllers for Linear Parameter-Varying Systems by the Gain-Scheduling Technique," Proceedings of the 29th IEEE Control Decision Conference, IEEE, New York, 1990, pp. 2490,2491 
${ }^{20}$ Apkarian, P., and Gahinet, P., "A Convex Characterization of GainScheduled $H_{\infty}$ Controllers," IEEE Transactions on Automatic Control, Vol. 40, No. 5, 1995, pp. 853-864.

${ }^{21}$ Wu, F., Packard, A., and Balas, G. J., "LPV Control Design for PitchAxis Missile Autopilots," Proceedings of the 34th IEEE Control Decision Conference, Inst. of Electrical and Electronics Engineers, New York, 1995, pp. 188-193.

${ }^{22}$ Wolodkin, G., Balas, G. J., and Garrard, W. L., "Application to Parameter-Dependent Robust Control Synthesis to Turbofan Engines," Proceedings of the AIAA Aerospace Conference, AIAA, Reston, VA, 1998.

${ }^{23}$ Fialho, I., Balas, G., Packard, A., Renfrow, J., and Mullaney, C., "Linear Fractional Transformation Control of the F-14 Aircraft Lateral-Directional
Axis During Powered Approach Landing," Proceedings of the American Control Conference, Vol. 1, IEEE, Piscataway, NJ, 1997, pp. 128-132.

${ }^{24}$ El Ghaoui, L., and Scorletti, G., "Control of Rational Systems Using Linear-Fractional Representations and Linear Matrix Inequalities," Automatica, Vol. 32, No. 9, 1996, pp. 1273-1284.

${ }^{25}$ Scorletti, G., "Some Results About the Stabilization and the $\mathcal{L}_{2}$-Gain Control of LFT Systems," Proceedings of the CESA'96, an IMACS-IEEE Multiconference on Computational Engineering in Systems Applications (CESA'96), Lille, France, July 1996.

${ }^{26}$ Blue, P., and Balas, G. J., "Linear Parameter-Varying Control for Active Flutter Suppression," AIAA 1997 Guidance, Navigation, and Control Conference, AIAA, Reston, VA, 1997. 